\title{
Healthy aging is associated with increased neural processing of positive valence but attenuated processing of emotional arousal: an fMRI study
}

\author{
Elizabeth G. Kehoe ${ }^{\mathrm{a}, \mathrm{b}, *}$, John M. Toomey ${ }^{\mathrm{c}}$, Joshua H. Balsters ${ }^{\mathrm{b}, \mathrm{d}}$, Arun L.W. Bokde ${ }^{\mathrm{a}, \mathrm{b}}$ \\ ${ }^{a}$ Cognitive Systems Group, Discipline of Psychiatry, School of Medicine, Trinity College Dublin, Dublin, Ireland \\ ${ }^{b}$ Trinity College Institute of Neuroscience, Trinity College Dublin, Dublin, Ireland \\ ${ }^{c}$ School of Computing, Dublin Institute of Technology, Dublin, Ireland \\ ${ }^{d}$ School of Psychology, Trinity College Dublin, Dublin, Ireland
}

Received 5 April 2012; received in revised form 3 July 2012; accepted 11 July 2012

\begin{abstract}
Arousal and valence play key roles in emotional perception, with normal aging leading to changes in the neural substrates supporting valence processing. The objective of this study was to investigate normal age-related changes in the neural substrates of emotional arousal processing. Twenty-three young and 23 older, healthy women underwent functional magnetic resonance imaging as they viewed images which were neutral or positive in valence and which varied in arousal level from low to high. Using a parametric modulation approach, we examined how the blood oxygen-level dependent signal varied with single trial subjective ratings of valence and arousal, and whether this differed with age. In accordance with previous studies we found that the older group showed greater activation in response to positive valence, in the left amygdala, left middle temporal gyrus and right lingual gyrus. In contrast however, they showed reduced reactivity to emotional arousal, in occipital and temporal visual cortices bilaterally, the left inferior parietal cortex, and the supplementary motor area bilaterally. This study represents the first of its kind to clearly dissociate how aging affects the neural correlates of emotional arousal and valence. The changes in arousal processing may in part be mediated by the functional reorganization evident in the aging brain, such as reduced activation of the posterior cortices as described by the posterior-anterior shift in ageing (PASA) effect.
\end{abstract}

(C) 2012 Elsevier Inc. All rights reserved.

Keywords: Aging; Emotion; fMRI; Arousal; Valence

\section{Introduction}

While a number of mental faculties decrease with age, emotional processing is well-preserved with healthy aging. Many older adults in fact enjoy improved emotional regulation and increased positive affect (Carstensen et al., 2003; Charles et al., 2001; Williams et al., 2006); and although cognitive control declines with age (Braver and Barch, 2002), emotional control does not, and may in fact improve (Gross et al., 1997). Older adults have been found to demonstrate a bias toward positive emotional stimuli and de-

\footnotetext{
* Corresponding author at: LloydBuilding, Trinity College Institute of Neuroscience, Trinity College Dublin, Dublin 2, Ireland. Tel.: +353 (0)1 896 4102; fax: +353 (0)1 8963183 .

E-mail address: elkehoe@tcd.ie (E. Kehoe).
}

creased processing of negative emotional stimuli, a finding that has been called "the positivity effect" (Mather and Carstensen, 2005). This positivity effect also appears to influence the retention of information, with older adults remembering more positive and less negative information than young people (Charles et al., 2003; Kennedy et al., 2004), and recalling autobiographical memories with more positivity (Comblain et al., 2005).

Functional magnetic resonance imaging (fMRI) studies have revealed significant age-related differences in the neural substrates of positive emotional valence, such as increased activation in response to positive versus neutral or negative stimuli in accordance with the positivity effect (Mather and Carstensen, 2005). For example, older adults showed greater amygdala activity to positive versus negative images on an emotional perception task (Mather et al., 
2004); while during the successful encoding of positive but not negative images, older adults showed greater activation in the medial prefrontal (mPFC) and the anterior cingulate gyrus compared with younger adults (Kensinger and Schacter, 2008). Increasing age has been found to be positively associated with activation in the mPFC to happy faces, but negatively associated with activation to sad faces in the same region (Williams et al., 2006).

While there have been numerous studies investigating age-related differences in emotional valence processing, there is a relative dearth of research into whether there are age-related differences in the processing of emotional arousal. Emotional arousal or intensity is thought to reflect the motivational value of an emotion (Lang et al., 1998), and has a strong effect on cognition, enhancing attention (Davis and Whalen, 2001; Fox et al., 2001) and also memory, via the increased activation of the amygdala and hippocampus (Cahill et al., 1996; Dolcos et al., 2004; Kensinger and Schacter, 2006; Richardson et al., 2004). There is some evidence that automatic arousal processing is relatively stable in old age, with older adults showing the same ability as young adults to detect high arousing stimuli (Leclerc and Kensinger, 2008), and threatening stimuli such as highly arousing negative scenes from the international affective picture system (IAPS) (Rösler et al., 2005), or threatening faces (Mather and Knight, 2006). There have been conflicting reports of older adults' autonomic response to emotional arousal however. Older adults have been found to show less increases in the skin conductance response (SCR) to highly-arousing negative images compared with younger adults, despite higher self-reports of arousal levels (Gavazzeni et al., 2008). This would imply that aging is associated with a decreased autonomic response to emotional arousal; however, other studies have contradicted this result (Denburg et al., 2003). Several behavioral studies have also reported age-related changes in arousal responses, with older adults rating positive emotional images as less arousing than young adults (Grühn and Scheibe, 2008; Pôrto et al., 2011).

As yet however, it is unknown whether the neural response to emotional arousal changes in healthy aging. The influence of aging on arousal processing is an important question given the central role that arousal plays in orienting attention (Vuilleumier, 2005), and in successful memory encoding (Sharot and Phelps, 2004). Because older adults tend to show decline on attention and memory tasks (Finnigan et al., 2011; Glisky, 2007) which benefit from moderately high levels of arousal (Bradley et al., 2003; McGaugh, 2006), altered arousal processing may play a pivotal role in this deterioration of function. This study therefore sought to clarify whether there are age-related differences in emotional arousal processing.

In the current study we investigated how healthy aging modulates the brain's response to the two emotional dimensions valence and arousal. A group of young and older healthy women viewed images which were either neutral or positive in emotional valence and which varied in arousal level as they underwent fMRI. Only women were included in the study because there have been considerable differences found between genders in emotional reactivity (Bradley et al., 2001) and in the neural representation of emotion (Beck et al., 1996; Cahill et al., 2001; Wager et al., 2003; Wrase et al., 2003). Similar to several other fMRI studies of emotional processing (Heinzel et al., 2005; Kehoe et al., 2011; Lewis et al., 2007; Phan et al., 2004), we utilized a parametric modulation approach, including the participants' subjective emotional ratings as parametric modulators in the fMRI analysis. This method allowed us to isolate the brain regions where the blood oxygen-level dependent (BOLD) response varied linearly with valence and arousal, and investigate whether this differed in the aging brain.

We predicted, given the findings of previous neuroimaging studies (Mather and Carstensen, 2005; Mather et al., 2004), that the older adults would show a greater BOLD response to positive emotional valence than the young adults, possibly in the amygdala and prefrontal cortex (PFC), in accordance with the predictions of the positivity effect. Given that it has previously been reported that older adults rate emotional images as less arousing (Grühn and Scheibe, 2008; Pôrto et al., 2011) and show a reduced SCR in response to arousal (Gavazzeni et al., 2008), we predicted that the older adults would show less BOLD modulation than the young group in response to increasing levels of arousal.

\section{Methods}

\subsection{Participants}

Twenty-three young (mean age $=23.04 \pm 3.48$ years; age range $=19-30$ years) and 23 older, healthy, righthanded women (mean age $=61.0 \pm 5.24$ years; age range $=55-71$ years) took part in this study. The young cohort is the same as those described in Kehoe et al. (2011); however this study additionally contains data from an older cohort and concentrates on aging differences. The groups did not differ in their educational attainment $(t=1.15, p>0.05)$. Depression scores on the Beck Depression Inventory (BDI-II; Beck et al., 1996) were $4.22 \pm 3.38$ and $3.73 \pm 3.60$ respectively, indicating an absence of depressive symptoms, and the scores did not differ statistically between the groups $(t=-0.34$, $p=0.74)$. We wished to exclude any participants with possible depression as the disorder is associated with significant alterations in emotional processing (Fales et al., 2008; Leppänen, 2006; Liu et al., 2011).

Personality was assessed using the Eysenck Personality Questionnaire, short-scale (EPQ-R) (Eysenck and Eysenck, 1991), and although not a focus of this study it is worth noting that while the groups did not differ in terms of extraversion levels, the young adults had on average higher 
neuroticism scores than the older adults $(t(44)=3.07, p=$ 0.004). Previous studies have found that neuroticism can influence the neural substrates of emotional processing (Canli et al., 2001; Haas et al., 2008; Kehoe et al., 2011), leading to sustained processing of sad stimuli for example (Haas et al., 2008), and increased activation in response to arousal in the mPFC (Kehoe et al., 2011). Therefore in order to control for the possible confounding effect of this trait it was included as a covariate of no interest in all of the fMRI analyses. The output was carefully examined, and the agerelated differences were not altered by the inclusion or exclusion of neuroticism in the model. Therefore we are confident that differences in this trait did not drive the age-related differences we report in this study.

In order to screen for possible subclinical dementia subtypes such as mild cognitive impairment (Petersen et al., 1999), all older adults underwent a neuropsychological assessment which included the Consortium to Establish a Registry for Alzheimer's Disease (CERAD) neuropsychological battery (Morris et al., 1988, 1989). The CERAD battery has been shown to be sensitive to the presence of age-related cognitive decline (Welsh et al., 1991, 1992) and therefore was used to detect possible cognitive impairment in the older adults. All of the older participants included in the study scored no more than 1.5 SD below the standardized mean scores for women of a similar age and education level on any of the subtests. The participants' demographic details and a subset of the neuropsychological scores are summarized in Table 1.

The study had full ethical approval from the St. James' Hospital and the Adelaide and Meath Hospital, incorporating the National Children's Hospital Research Ethics Committee. All of the participants gave written informed consent before commencing the study. The young participants were recruited from Trinity College, Dublin, with the use of poster advertisements. The older participants

Table 1

Summary of participant demographic characteristics and neuropsychological test scores

\begin{tabular}{lccll}
\hline & Young & Older & Group difference & $p$ \\
\hline Age (y) & $23.04(3.46)$ & $61(5.24)$ & $t(44)=-28.99$ & $<0.001$ \\
Education (y) & $18.09(2.52)$ & $16.83(4.6)$ & $t(44)=1.15$ & 0.255 \\
BDI & $4.22(3.38)$ & $3.73(3.6)$ & $t(44)=-0.34$ & 0.735 \\
EPQ-N & $4.83(2.62)$ & $2.57(2.37)$ & $t(44)=3.07$ & 0.004 \\
EPQ-E & $8(3.58)$ & $8.3(2.8)$ & $t(44)=-0.32$ & 0.75 \\
EPQ-P & $2.22(1.44)$ & $2.04(1.61)$ & $t(44)=0.386$ & 0.702 \\
MMSE & - & $29.3(0.97)$ & - & - \\
NART & - & $40(4.72)$ & - & - \\
\hline
\end{tabular}

Standard deviations are indicated in parentheses. The $p$-values are from a series of independent $t$ tests which examined between-group differences. Key: BDI, Beck Depression Inventory; extraversion scores; EPQ-E, Eysenck Personality Questionnaire; EPQ-N, Eysenck Personality Questionnaire, neuroticism scores; EPQ-P, Eysenck Personality Questionnaire; MMSE, Mini-Mental State Examination (included in the Consortium to Establish a Registry for Alzheimer's Disease (CERAD) neuropsychological battery); NART, National Adult Reading Test (included in the CERAD neuropsychological battery). were recruited from the greater Dublin area through advertisements in church newsletters and volunteering Web sites.

\subsection{Stimulus set}

The stimuli for the fMRI paradigm consisted of 190 colored photographs as described in detail in Kehoe et al. (2011), which were either positive or neutral in valence and varied in arousal level over the entire range. They were a combination of 98 images from the IAPS (Lang et al., 2008) and 92 images gathered by the experimenters from various sources. In order to control for the interaction of arousal and valence, as these 2 dimensions tend to be correlated (Lang et al., 2008), equal numbers of positive low arousal, positive higher arousal, neutral low arousal, and neutral higher arousal stimuli were included. It was hoped that this would allow for the dissociation of the effects of arousal and valence on the BOLD signal. The valence dimension was also limited to neutral and positive so that linear increases in the BOLD signal due to valence could be ascribed to increasing positivity, without being confounded by the inclusion of negative stimuli.

\section{3. fMRI paradigm}

The task was an emotional perception task (Kehoe et al., 2011), in which the participants viewed 190 colored images which varied in valence and arousal as they underwent fMRI. The task consisted of 2 experimental runs, each containing 95 trials and lasting approximately 20 minutes. In each trial an image was presented in the center of a white background for $3000 \mathrm{~ms}$, and after a delay of 1000 to 3000 ms (pseudorandom jitter), a prompt appeared on screen for $2000 \mathrm{~ms}$ asking the participants to classify the image they had just seen as "Living" or "Nonliving" (Fig. 1). The participants were instructed to make their response by pressing either the left or right button on a magnetic resonancecompatible button response box held in their right hand, to correspond with the left/right position of the "Living/Nonliving" word on the screen. The task was intended to maintain the participants' focus for the duration of the task, without explicitly drawing their attention to the emotional content of the stimuli (Kensinger et al., 2007). The stimuli were pseudorandomized so that no more than 3 images of the same valence or arousal type were presented in a row, in order to avoid inducing long-lasting mood states. The stimuli were delivered using Presentation version 13.0 (Neurobehavioral Systems, Albany, CA, USA).

\subsection{Postscanning image rating}

The participants did not rate the images while in the scanner as the processes of re-evaluation and judgment have been found to attenuate the neural response to emotional stimuli (Hariri et al., 2000; Taylor et al., 2003). Instead they returned 2 to 3 days later and rated all of the images they had seen during the scanning session as well as 48 negative 


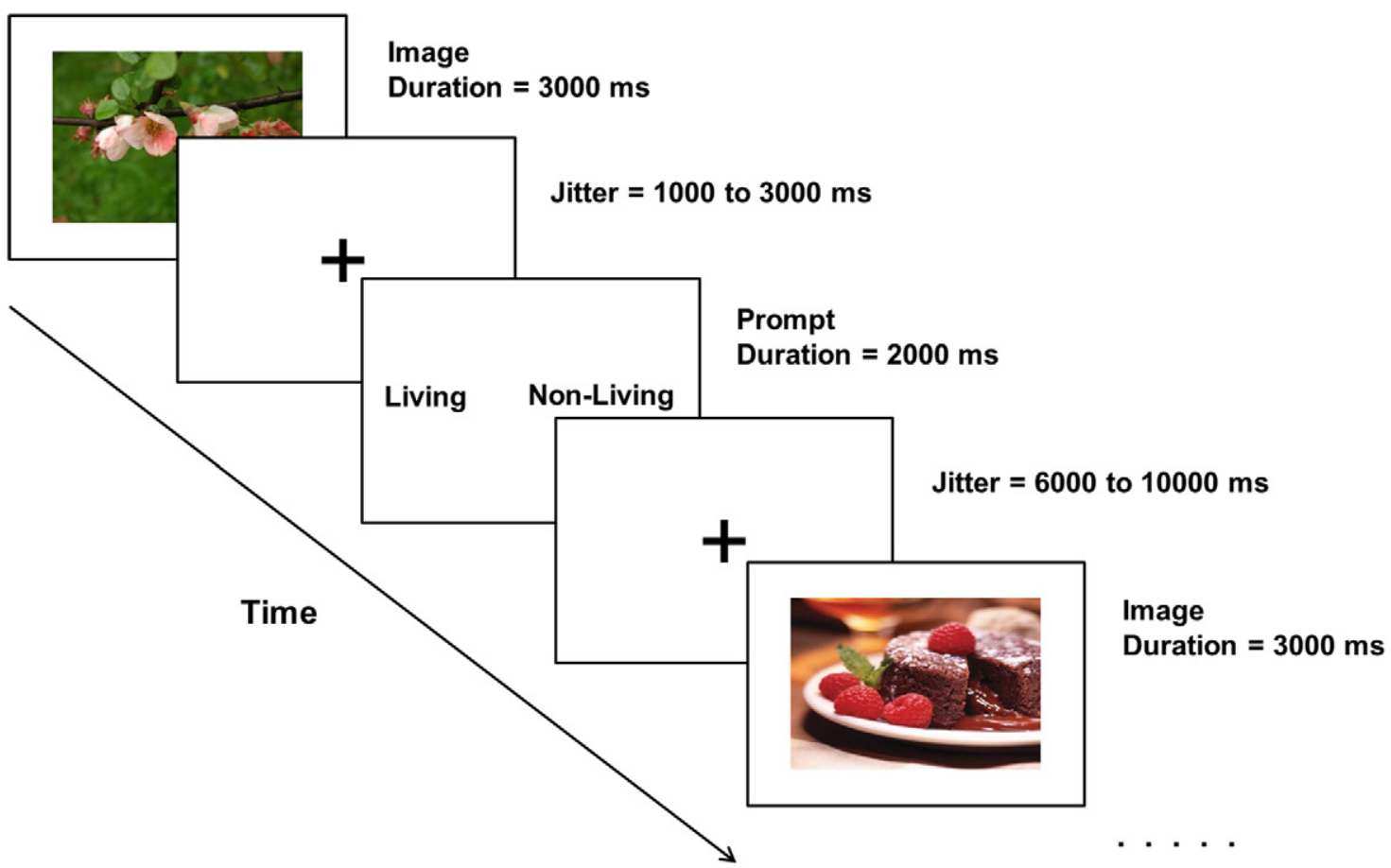

Fig. 1. Schema of the emotional perception paradigm. In each trial, an image appeared on screen for $3000 \mathrm{~ms}$, and following a jittered delay of 1000-3000 ms a prompt appeared asking the participant to classify the image they had just seen as living or nonliving. The intertrial interval was between 6 and 10 seconds.

images from the IAPS along the dimensions of valence, arousal, and dominance. The negative images were included in the rating task to provide contrast with the others, to ensure that the participants understood the full remit of the valence dimension. A computerized version of the SelfAssessment Manikin (Lang et al., 2008) was used to operationalize valence, arousal, and dominance. This study focused on the effects of arousal and valence only. The valence dimension ranged from 1 to 9 , where 1 represented the most extreme negative rating, 5 was neutral and 9 was the most pleasant rating. Along the arousal dimension 1 represented the lowest arousal rating while 9 was the most arousing and intense.

\subsection{Magnetic resonance imaging scanning protocol}

Imaging data were acquired using a Philips Intera Achieva 3.0 T MR system (Best, The Netherlands). The BOLD signal changes were measured using a $\mathrm{T} 2 *$-weighted echo-planar imaging sequence with repetition time (TR) = $2000 \mathrm{~ms}$ and echo time (TE) $=30 \mathrm{~ms}$. Each volume of data covered the entire brain with 39 slices, and the slices were acquired in interleaved sequence from inferior to superior direction. Five hundred ninety-eight volumes were acquired during each experimental run, with voxel dimensions of $3.5 \times 3.5 \times 3.85 \mathrm{~mm}$ and a $0.35 \mathrm{~mm}$ gap between the slices. A T1-weighted/inversion recovery (T1W/IR) sequence was used to collect a 3-D highresolution anatomical image with voxel dimensions equal to $0.9 \times 0.9 \times 0.9 \mathrm{~mm}$ for structural localization.

\subsection{Behavioral data analysis}

The experimental log files were parsed using Python scripts (Version 2.6.2, www.python.org) to extract performance and rating information. These data were then used to create individually tailored regressors for each participant based on their subjective ratings of the images. Statistical analyses of the behavioral results were conducted using IBM SPSS Statistics v.19 (Armonk, NY, USA). The young and older groups' mean ratings of the emotional images were compared with a series of 5 independent sample $t$ tests, and a Bonferroni correction for multiple comparisons was applied.

\subsection{Magnetic resonance imaging data analysis}

The magnetic resonance imaging data were analyzed using AFNI (Cox, 1996) (afni.nimh.nih.gov/afni) and FSL (FMRIB Software Library; www.fmrib.ox.ac.uk/fsl). The data were motion corrected by realignment to the first volume of the first run, concatenated into a single run, global mean-adjusted by proportional scaling and smoothed with a $6 \mathrm{~mm}$ full-width-at-half-maximum Gaussian kernel. A general linear model analysis was conducted in AFNI. Two regressors modeled the mean BOLD signal change from baseline during the image and prompt trials. To model the additional effects of arousal and valence on the BOLD response during the image trials, subjective ratings of arousal and valence for each image were included as single trial parametric weights. This resulted in 3 image regressors: 
a constant unmodulated regressor describing the mean BOLD response during the image presentations, independent of the arousal or valence of the image (this will be referred to as the constant BOLD response), BOLD activation during the image trials modulated by arousal, and BOLD activation during the image trials modulated by valence. A separate regressor was included for any of the images rated as negative, so that these trials were not included in the amplitude modulation analysis. Six motion parameters, 8 regressors to model low frequency noise, and 2 regressors to model the mean differences between the 2 runs were also included as regressors of no interest in the general linear model.

All statistical analyses were calculated in the participants' native space, then the results were warped to the Montreal Neurological Institute (MNI) brain template (Montreal Neurological Institute/International Consortium for Brain Mapping 152 standard atlas as provided in the FSL software package) using FSL's linear registration tool, FLIRT. The transformation matrix (12 parameter affine) from native space to MNI space was calculated using the high resolution structural images from each subject. In order to confirm that the images were not flipped during the coregistration process, the activation maps were checked for contralateral motor activity timelocked to responses in the prompt conditions when they were warped into MNI space.

The group statistical analyses were based on a randomeffects model, and activation maps of the constant and modulated responses were calculated for the young and older groups with a series of independent $t$ tests. The groups' activation maps were then compared with a series of analyses of covariance, which controlled for neuroticism scores, which differed significantly between the groups. Neuroticism has previously been found to influence the neural substrates of emotional processing (Canli, 2004; Cremers et al., 2010; Kehoe et al., 2011), therefore we wished to control for possible confounding effects which might mask age-related differences.

Statistically significant clusters passed a voxelwise statistical threshold of $p \leq 0.01$; and a minimum cluster size of $708 \mu \mathrm{L}$ of contiguous statistically significant voxels was applied to correct for multiple comparisons across the brain.
This cluster size was calculated using a Monte Carlo simulation to obtain a family-wise error corrected $p<0.05$ statistical significance in the $t$ tests. A small volume correction was used for the amygdala and hippocampus, with a minimum cluster size for these structures of $94 \mu \mathrm{L}$ of contiguous statistically significant voxels (approximately 12 voxels). This value was calculated by applying a Monte Carlo simulation to masks created for the amygdala and hippocampus using the FSL Harvard-Oxford subcortical structural atlas. The Statistical Parametric Mapping (SPM8, http://www.fil.ion.ucl.ac.uk/spm) anatomy toolbox (V1.7b, Eicknoff et al., 2005) was used to localize activation clusters; however where there were no probabilistic cytoarchitectonic labels available a Brodmann area is given in the results table instead.

\section{Results}

\subsection{Emotional ratings}

The average ratings of the stimuli by the young and older groups were calculated in order to examine the overall pattern of age-related differences in emotional ratings ( $\mathrm{Ta}-$ ble 2). However it should be noted that subjective rather than average ratings were used for all of the fMRI analyses. The older group on average rated more images as positive than the young group $(t(44)=-3.61, p=0.001)$, while the young group rated more images as highly arousing than the older group $(t(44)=2.83, p=0.007)$ (see Table 2$)$. The amplitude modulation approach used to model the effects of arousal and valence on the BOLD signal is not dependent upon the total number of images per category, as it is based on linear association. Therefore we do not expect the fMRI results to be biased by these differences in behavioral ratings. In order to assess whether there was a correlation between the arousal and valence of the images, a correlation analysis was performed on the average ratings of the images from the fMRI task given by both the young and older groups. There was a statistically significant negative correlation between the average valence and arousal rating of the stimuli for both the young $(r=-.24, p=0.001)$ and older groups $(r=-.43, p<0.001)$.

Table 2

Average ratings of the emotional images from the young and older adults

\begin{tabular}{|c|c|c|c|c|c|}
\hline & Positive & Neutral & Negative & High arousal & Low arousal \\
\hline Young & $53.87(26.91)$ & 113.04 (37.19) & $18.43(13.96)$ & $67.35(16.74)$ & 99.57 (13.76) \\
\hline Old & $84.13(29.88)$ & 77.30 (34.46) & $25.39(12.68)$ & $51.17(21.74)$ & $110.26(23.48)$ \\
\hline $\operatorname{Sig} p^{\mathrm{a}}$ & Sig** $(0.001)$ & Sig** (0.002) & NS $(0.084)$ & Sig** (0.007) & NS $(0.066)$ \\
\hline
\end{tabular}

Note that these are for guidance only as the functional magnetic resonance image (fMRI) analysis was based on subjective, not average ratings. The scores represent the total number of stimuli in the emotional perception task that the groups rated as positive, neutral, or negative in valence; and lower or higher in arousal. The arousal categories include only positive and neutral images as negative were excluded from the fMRI analysis. Positive: valence $=7-9$; Neutral: valence $=4-6$; Negative: valence $=1-3 ;$ High arousal: arousal $=6-9 ;$ Low arousal: arousal $=1-5$.

Key: NS, not significant. ** $p<0.01$.

${ }^{a}$ Indicates the results from a series of $t$ tests (corrected with a Bonferroni correction for multiple comparisons) which examined group differences. 
Table 3

Age-related differences in the valence-dependent BOLD response

\begin{tabular}{lccc}
\hline & Voxels, $n$ & $t$ Statistic & MNI coords $\mathrm{x}, \mathrm{y}, \mathrm{z}$ \\
\hline Older $>$ young & & & \\
Right lingual gyrus & 123 & 4.49 & $28,-90,-14$ \\
Left middle temporal gyrus & 111 & 3.60 & $-54,-66,4$ \\
Left amygdala & 26 & 3.85 & $-24,-2,-26$ \\
\hline
\end{tabular}

Montreal Neurological Institute (MNI) coordinates are - LPI (i.e., negative values indicate left, posterior, and inferior directions). Statistical significance level of t-values: $t \geq 2.82, p<0.01 ; t \geq 3.79, p<0.001$, corrected.

Key: BOLD, blood oxygen-level dependent.

${ }^{a}$ Labels were generated using the Statistical Parametric Mapping (SPM8, http://www.fil.ion.ucl.ac.uk/spm) anatomy toolbox (V1.7b, Eicknoff et al., 2005); however where there was no cytoarchitectonic information available a Brodmann area (BA) is given.

\section{2. fMRI results}

\subsubsection{Dissociating the effects of arousal and valence in the young and older adults}

The young and older adults' constant and arousal and valence-dependent BOLD responses were first analyzed separately, in order to examine whether arousal and valance were processed by distinct neural networks, or whether their responses overlapped in the brain. The results from the young group are described in detail in Kehoe et al. (2011), however to recapitulate briefly, there were arousal-dependent BOLD increases bilaterally in the fusiform gyrus, middle temporal gyrus, calcarine gyrus, middle occipital gyrus, thalamus, middle cingulate cortex, and inferior frontal gyrus; while the valencedependent response was confined to a region of the left fusiform gyrus. In the older adults the arousal-dependent BOLD increase was much less widespread than in the young group, confined to a region of the right middle occipital gyrus; while valence-dependent BOLD increases were observed in regions of the right middle occipital gyrus, the right calcarine gyrus, and the right fusiform gyrus. A single region in the right middle occipital gyrus was modulated by both arousal and valence.

Independent of arousal or valence level, both groups showed widespread activation in the constant response to the images, in the occipital and temporal cortices (e.g., calcarine gyrus, middle occipital gyrus, fusiform gyrus, inferior, middle, and superior temporal gyri), the hippocampus, the middle frontal gyrus, the parietal lobe, and the cerebellum. The interested reader is referred to Supplementary Figs1 and 2. which accompany this article.

\subsubsection{Age-related differences in the valence-dependent $B O L D$ response}

When the young and older adults' results were directly compared, the older group showed a significantly greater valence-dependent BOLD response to positive valence, consistent with the "positivity effect" in aging. This effect was evident in 3 clusters, located in the left amygdala, the right lingual gyrus, and the left middle temporal gyrus (Table 3, Fig. 2).

\subsubsection{Age-related differences in the arousal-dependent $B O L D$ response}

The young group displayed a significantly greater arousal-dependent BOLD response than the older adults in a number of brain regions. These included the occipital and temporal visual processing regions bilaterally, the right angular gyrus, the left inferior parietal cortex, the supplementary motor area (SMA), the left superior medial gyrus, and the right middle cingulate cortex. The older group showed a greater arousal-dependent response than the young group in 1 region however, in the right middle frontal gyrus (MFG), Brodmann area 10 (Table 4, Fig. 3).

\subsubsection{Age-related differences in the constant BOLD response}

The young group displayed significantly more activation in the constant BOLD response than the older group in a wide range of brain areas (Table 5, Fig. 4a and b). These included visual processing areas spanning the occipital and temporal lobes, including primary and secondary visual cortex, the inferior, middle, and superior temporal gyri, and the fusiform gyrus. The young group also displayed greater constant BOLD activation in the parietal lobe, the hippocampus, and putamen bilaterally and the cerebellum. The older group did display greater constant BOLD activation than the young group in a single region however, in the right supramarginal gyrus (see Table 5, and Fig. 4c).

\section{Discussion}

The results of the current study suggest that the neural substrates of positive valence and emotional arousal processing are significantly altered by healthy aging. Using a parametric modulation paradigm and subjective emotional ratings, we identified 2 main differences in emotional valence and arousal processing with age. Both the behavioral ratings and the fMRI results strongly support the positivity effect in aging. The older adults on average rated more images as positive than the young adults, and they also demonstrated a stronger modulation of the BOLD signal with increasing levels of positive valence in several brain regions, including the left amygdala, the left middle temporal gyrus (MTG), and the right lingual gyrus. Other fMRI 
(a) $\mathrm{R}$

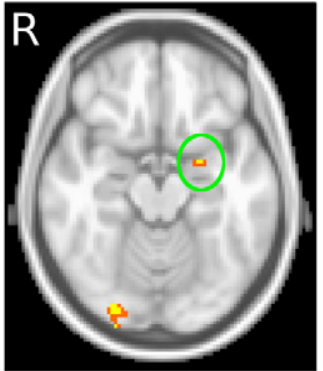

$-24,-2,-26 \mathrm{~mm}$

(b) $R$

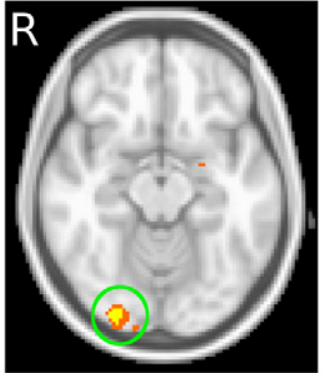

$28,-90,-14 \mathrm{~mm}$

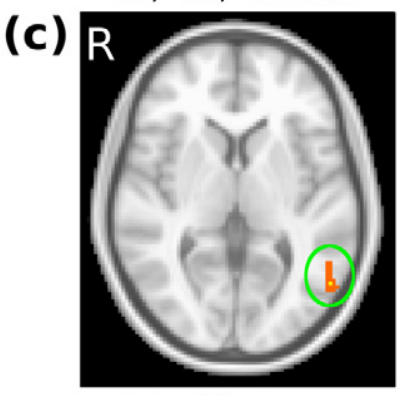

$-54,-66,4 \mathrm{~mm}$
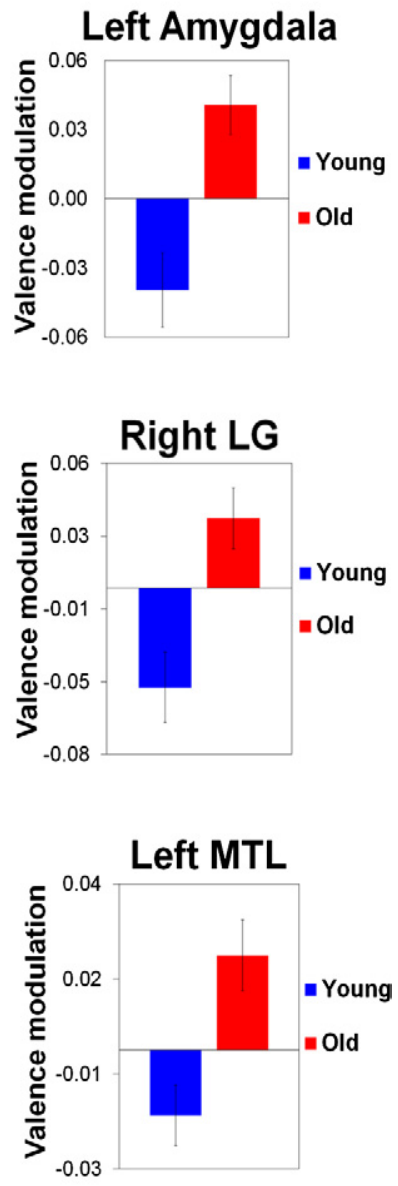

Fig. 2. Age-related differences in the valence-dependent blood oxygenlevel dependent (BOLD) response. The older group displayed significantly greater BOLD modulation due to positive valence than the young group in the (a) left amygdala, (b) right lingual gyrus and (c) left middle temporal gyrus. The images on the left are results from an analysis of covariance comparing the young and older groups' valence-dependent BOLD signal, and the green circles indicate the clusters of difference. The bar charts show the average signal for the young and older groups at the peak voxel in each cluster, with standard errors denoted by the error bars.

studies have also found similar results, with older adults showing increased BOLD signal in the amygdala compared with young adults in response to positive emotional images (e.g., Mather et al., 2004). This increased processing of positive valence in older adults may reflect a greater emphasis on emotional wellbeing, as predicted by the socioemotional selectivity theory (Carstensen et al., 2003). According to this theory, as people age and time becomes more limited they prioritize their emotional wellbeing, engaging in more emotional regulation, and experiencing more positive emotions. These increases in activation also indicate however that the reduced BOLD signal in the older group in response to arousal and in the constant response, are likely not simply driven by reduced hemodynamic functioning caused by changes in arterial elasticity or cortical atrophy with increasing age. Rather, they represent genuine agerelated changes in the neural activation associated with emotional processing.

In contrast to the valence results, the young group both rated the emotional images as more arousing, and also demonstrated a greater BOLD signal increase in response to arousal in a number of cortical regions. These included the occipital and temporal cortices, including early visual cortex and the middle and inferior temporal gyri; as well as the left superior medial gyrus, the left supplementary motor area (SMA) and the middle cingulate cortex. This is the first study to our knowledge to demonstrate reduced cortical reactivity to emotional arousal in healthy aging. Aging has been associated with the experience of fewer negative emotions (Gruenewald et al., 2008; Mroczek and Kolarz, 1998), as well as reduced neural processing of negative compared with positive emotional stimuli (Addis et al., 2010; Leclerc and Kensinger, 2008; Mather et al., 2004). Considering that negative emotional stimuli tend to be rated as more arousing than positive stimuli (Lang et al., 2008), and tend also to result in greater BOLD signal increases in emotional and visual brain regions than neutral or positive images (e.g., Lane et al., 1997; Mourão-Miranda et al., 2003), it is possible, based on our results, that an under-responsiveness in older adults to the effects of arousal may partly mediate the attenuation in their response to negative emotional stimuli. It may be the case that changes in emotional processing in aging are marked by both increased sensitivity to positive valence, and attenuated arousal processing, which combine to produce the behavioral and neural patterns that are often observed, such as the positivity effect. Future studies could focus on disentangling these 2 mechanisms in order to determine whether the positivity effect is more likely due to the prioritization of emotional goals, a lessening of the impact of highly arousing emotional stimuli, or a combination of these 2 factors.

In the current study the older group did demonstrate a greater BOLD response to emotional arousal in a single region, located in the right MFG. This may indicate greater top-down, emotional control in the older adults, indicative of increased emotional regulation during the perception of highly arousing stimuli. Several previous neuroimaging studies have found that older adults display greater PFC activation associated with emotional regulation, in particular to negative emotional stimuli (Murty et al., 2009; St. Jacques et al., 2009; Williams et al., 2006). Further, given that negative images tend to be rated as more arousing than either neutral or positive ones (Lang et al., 2008), it may be that the effects of highly arousing stimuli are controlled by increased PFC regulation with age. These possibilities require further investigation with future studies, for example by including negative emotional stimuli which vary in arousal level; but understanding how arousal processing changes with healthy aging may provide insights into both emotional and cognitive changes with age. 
Table 4

Age-related differences in the arousal-dependent BOLD response

\begin{tabular}{|c|c|c|c|c|}
\hline & Voxels, $n$ & $t$ Statistic & MNI coords $\mathrm{x}, \mathrm{y}, \mathrm{z}$ & Area $^{a}$ \\
\hline \multicolumn{5}{|l|}{ Young $>$ older } \\
\hline Right middle temporal gyrus & 1163 & -4.24 & $44,-56,12$ & IPC(PGp): $20 \%$ \\
\hline Left calcarine gyrus & 427 & -4.30 & $-2,-68,14$ & Area 18: $10 \%$ \\
\hline Left middle occipital gyrus & 111 & -3.78 & $-28,-98,-4$ & V3v: $40 \%$ \\
\hline Right calcarine gyrus & 78 & -4.12 & $18,-102,-2$ & Area 17: $90 \%$ \\
\hline Left inferior temporal gyrus & 156 & -3.88 & $-60,-52,-16$ & BA 37 \\
\hline Left middle temporal gyrus & 123 & -3.48 & $-46,-68,8$ & hOC5(V5): $10 \%$ \\
\hline Right angular gyrus & 116 & -4.00 & $56,-64$ & IPC (PGp): $80 \%$ \\
\hline Left inferior parietal cortex & 133 & -4.09 & $-52,-72,26$ & IPC(PGp): $70 \%$ \\
\hline Left SMA & 200 & -4.43 & $-2,-6,72$ & Area 6: $70 \%$ \\
\hline Left superior medial gyrus & 122 & -4.58 & $-6,64,8$ & BA 10 \\
\hline Right middle cingulate cortex & 99 & -3.72 & $2,-8,40$ & Area 6: $20 \%$ \\
\hline \multicolumn{5}{|l|}{ Older $>$ young } \\
\hline Right middle frontal gyrus & 141 & 4.06 & $36,42,14$ & BA10 \\
\hline
\end{tabular}

Montreal Neurological Coordinates (MNI) coordinates are -LPI (i.e., negative values indicate left, posterior and inferior directions). Statistical significance level of t-values: $t \geq 2.82, p<0.01 ; t \geq 3.79, p<0.001$, corrected.

Key: BOLD, blood oxygen-level dependent; SMA, supplementary motor area.

a Labels were generated using the Statistical Parametric Mapping (SPM8, http://www.fil.ion.ucl.ac.uk/spm) anatomy toolbox (V1.7b, Eicknoff et al., 2005); however where there was no cytoarchitectonic information available a Brodmann area (BA) is given.

(a) $\mathrm{R}$

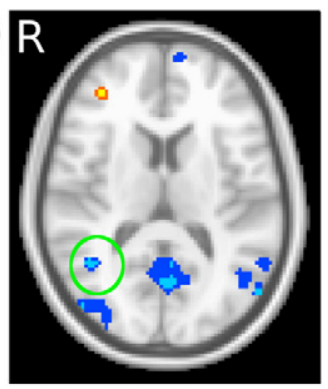

$44,-56,12 \mathrm{~mm}$

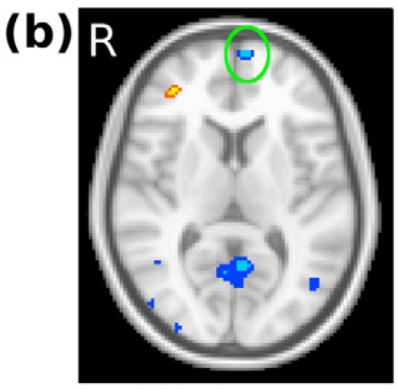

$-6,64,8 \mathrm{~mm}$

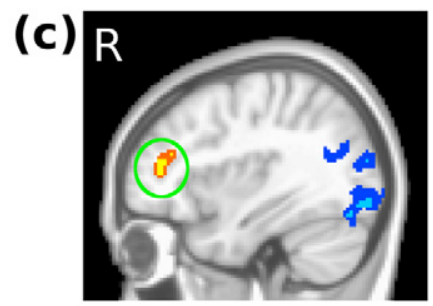

$36,42,14 \mathrm{~mm}$

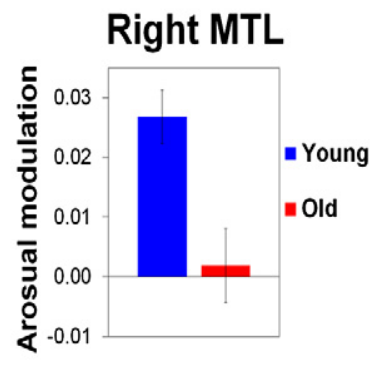

Left mPFC
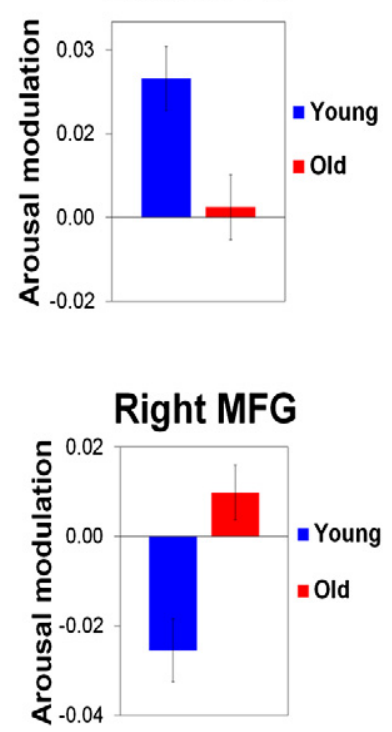

Fig. 3. Age-related differences in the arousal-dependent blood oxygenlevel dependent (BOLD) response. The young group showed significantly greater BOLD modulation due to arousal than the older group in several regions including the (a) right middle temporal gyrus and (b) the left medial prefrontal (mPFC); while the older group showed greater arousaldependent modulation in the (c) right middle frontal gyrus. See Fig. 2.

Independent of the emotional arousal or valence levels of the stimuli, the young group displayed significantly more constant BOLD activation while viewing the images than the old group in ventral visual processing areas (Haxby et al., 1991; Ishai et al., 1999; Pietrini et al., 2004), such as primary and secondary visual cortex bilaterally, and the inferior, middle and superior temporal gyri, and in the hippocampus. The older group showed greater activation in the right intraparietal area 1 (hIP1) however, an area that has been implicated in a wide variety of visuospatial and visuomotor functions (Choi et al., 2006). Previous aging studies have also found greater parietal activation in older adults when they perform as well as young adults on memory (Anderson et al., 2000) and perceptual tasks (Davis et al., 2008).

The age-related differences in both the arousal-dependent and constant BOLD responses parallel the posterioranterior shift in ageing (PASA) pattern which has been observed elsewhere (e.g., Dennis et al., 2007; Grady et al., 2002; Grossman et al., 2002), and which is not restricted to emotional processing (for a review see Davis et al., 2008). The PASA effect is seen when older adults demonstrate reduced occipitotemporal brain activation coupled with increased frontal or parietal activation compared with young adults, when performance is matched between the groups. As such the increased activation in the older adults in the right hIP1 in the constant response, and in the right MFG in response to arousal might be seen as compensatory mechanisms for the marked decrease in sensory processing in the visual and temporal cortices. Within this context differences in emotional processing in aging, including the reduced modulation of visual and temporal cortices by arousal, may be mediated as least in part, by functional reorganization and compensation which occurs in the aging brain, such as those described by the PASA effect (Davis et al., 2008). It 
Table 5

Age-related differences in the constant BOLD response

\begin{tabular}{|c|c|c|c|c|}
\hline & Voxels, $n$ & $t$ Statistic & MNI coords $\mathrm{x}, \mathrm{y}, \mathrm{z}$ & Area $^{a}$ \\
\hline \multicolumn{5}{|l|}{ Young $>$ older } \\
\hline Left calcarine gyrus & 4132 & -5.98 & $-16,-76,12$ & Area 17: $50 \%$ \\
\hline Left lingual gyrus & 447 & -3.88 & $-32,-86,-18$ & V3v: 30\%; V4: $30 \%$ \\
\hline Right inferior occipital gyrus & 111 & -3.97 & $40,-80,-8$ & V4: $30 \%$ \\
\hline Left precuneus & 1122 & -4.91 & $-12,-38,70$ & Area 4a: $40 \%$ \\
\hline Left insula lobe & 919 & -4.09 & $-40,-10,10$ & Insula (Ig2): $20 \%$ \\
\hline Right inferior temporal gyrus & 531 & -5.52 & $44,-44,-12$ & BA 20 \\
\hline Right heschls gyrus & 194 & -4.49 & $42,-20,12$ & Op1: $60 \%$; Op2: $40 \%$ \\
\hline Left temporal pole & 105 & -4.67 & $-44,12,-22$ & BA 38 \\
\hline Right superior temporal gyrus & 94 & -3.94 & $68,-12,-2$ & BA 22 \\
\hline Right superior temporal gyrus & 92 & -3.48 & $50,-4,-2$ & BA 52 \\
\hline Left putamen & 643 & -4.49 & $-28,-8,-4$ & - \\
\hline Right putamen & 610 & -5.65 & $32,8,4$ & - \\
\hline Right hippocampus & 201 & -4.49 & $16,-32,-6$ & Hipp(SUB): $80 \%$ \\
\hline Left hippocampus & 140 & -5.22 & $-20,-28,-8$ & Hipp(SUB): $50 \%$ \\
\hline Right cerebellum & 637 & -5.22 & $22,-56,-42$ & Cerebellar tonsil \\
\hline Cerebellar vermis & 222 & -5.37 & $2,-40,-14$ & Lobules I-IV: $90 \%$ \\
\hline \multicolumn{5}{|l|}{ Older $>$ young } \\
\hline Right supramarginal gyrus & 111 & 3.75 & $44,-40,38$ & hIP1: $20 \%$ \\
\hline
\end{tabular}

Montreal Neurological Institute (MNI) coordinates are -LPI (i.e., negative values indicate left, posterior and inferior directions). Statistical significance level of t-values: $t \geq 2.82, p<0.01 ; t \geq 3.79, p<0.001$, corrected.

Key: BOLD, blood oxygen-level dependent.

${ }^{\text {a }}$ Labels were generated using the Statistical Parametric Mapping (SPM8, http://www.fil.ion.ucl.ac.uk/spm) anatomy toolbox (V1.7b, Eicknoff et al., 2005); however where there was no cytoarchitectonic information available a Brodmann area (BA) is given.

remains to be resolved therefore whether age-related changes in the neural substrates of emotional processing are due to differences in emotional regulation processes and emotional prioritization, or whether they are indicative of widespread neural reorganization in the aging brain.

Although we did not measure autonomic responses to emotional arousal in the current study, we did measure subjective levels of arousal for every stimulus, and used these self-report measures as parametric modulators in our analysis of the fMRI data. This is an important improvement on previous studies of emotional processing in aging, as the results of this study and others (Grühn and Scheibe, 2008; Pôrto et al., 2011) indicate that aging significantly influences emotional ratings. The use of subjective ratings in the current paradigm exposed significant differences in emotional responses which may have been lost had average or standard ratings been used instead. A possible future study could measure autonomic arousal responses, such as SCR or pupil dilation (Gabay et al., 2011; O'Neill and Zimmerman, 2000), and examine their influence on the BOLD signal. These metrics could provide objective and physiological measures of arousal, to see whether age-related differences in the neural response to arousal are related to differences in autonomic arousal responses.

While the results of this and many other studies (Knight et al., 2007; Kwon et al., 2009; Mather and Carstensen, 2005 ) indicate a positivity bias in older adults, this effect is not always seen (e.g., Grühn et al., 2005; Isaacowitz et al., 2006). The emergence of the positivity effect can be reversed when attentional resources become limited for example (Knight et al., 2007), perhaps indicating that emo- tional goals are prioritized only when there are enough attentional resources available. The task in the current study was a passive emotional viewing paradigm however, which allowed full processing of the emotional stimuli, with no cognitive demands placed on the participants other than the simple shallow encoding task. This may have facilitated the emergence of the positivity effect. Further, other studies may have failed to identify an age-related positivity bias due to the inclusion of emotional stimuli on the basis of valence ratings made by young adults. This once again highlights the importance of taking into account individual differences in emotional ratings.

Several previous studies have indicated that arousal and valence may be processed by different neural systems (Kensinger and Corkin, 2004; Lewis et al., 2007; Mourão-Miranda et al., 2003); however their effects are difficult to dissociate experimentally as they tend to covary, especially in the case of negative stimuli (Lang et al., 2008). In the current study we endeavored to address this methodological constraint by including equal numbers of positive low arousal, positive higher arousal, neutral low arousal, and neutral higher arousal stimuli. This was intended to control for the interaction of arousal and valence from the outset of the experiment, so that their effects on brain activation could be dissociated. The behavioral ratings however revealed that there was a statistically significant correlation between the average valence and arousal ratings of the stimuli in both the young and older groups, albeit a negative correlation. This result was somewhat unexpected, given that previous studies have suggested that arousal and positive va- 
(a)

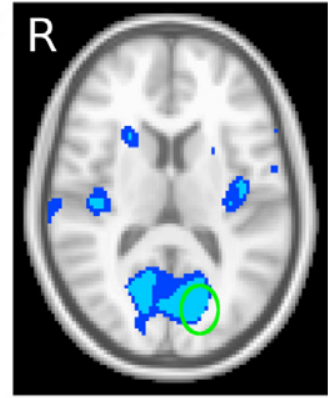

$-16,-72,12 \mathrm{~mm}$

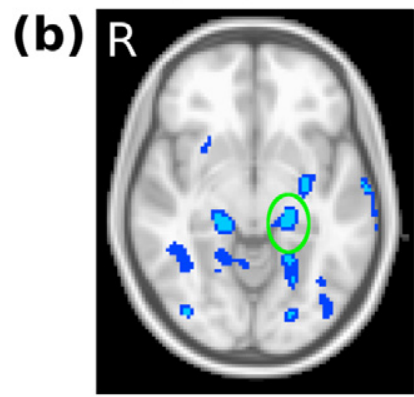

$-20,-28,-8 \mathrm{~mm}$

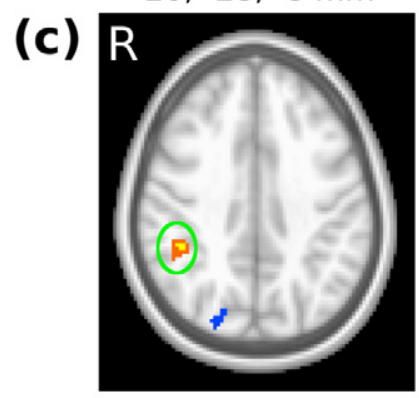

$44,-40,38 \mathrm{~mm}$
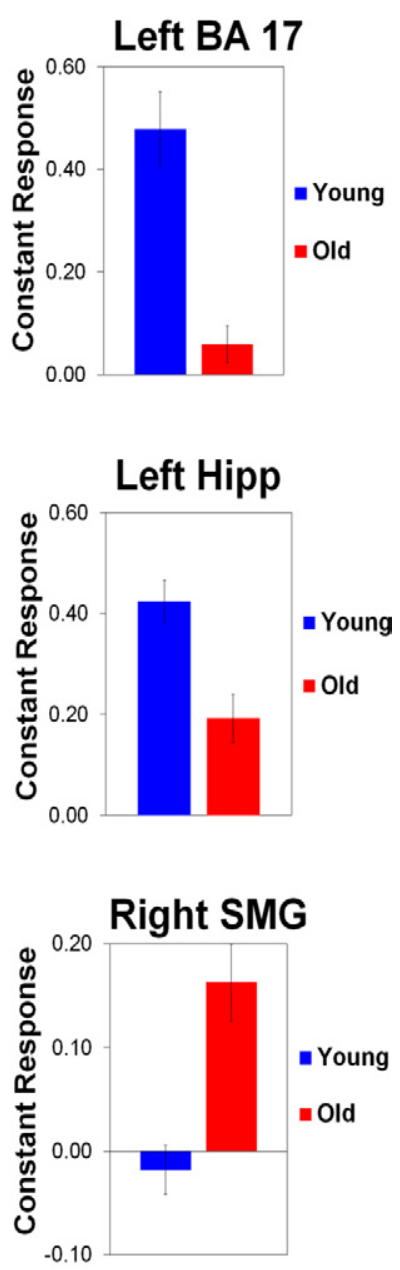

Fig. 4. Age-related differences in the constant blood oxygen-level dependent (BOLD) response. The young group showed significantly more activation in a number of regions including the (a) left calcarine gyrus and (b) left hippocampus; while the older group showed more activation in the (c) right supramarginal gyrus. See Fig. 2.

lence tend to be positively correlated (Lang et al., 2008); however the negative relationship we observed may be due in part to the inclusion of many low arousal positive valence images and neutral higher arousal images, which was intended to control for the covariation of arousal and valence. This correlation highlights the inherent difficulty in experimentally dissociating the effects of arousal and valence.

Despite this limitation however, the fMRI results indicated that in the young group the linear modulations of the BOLD signal due to arousal and valence were dissociable, with no brain region showing an increase in activation due to both arousal and valence (see Kehoe et al., 2011, and Supplementary Fig. 1). Examining the results of the same analysis for the older adults revealed that although the effects of arousal and valence were partially dissociable, there was a region in the right middle occipital gyrus that showed a BOLD signal increase to both dimensions (Supplementary Fig. 2). These results indicate that in the current paradigm the effects of arousal and valence on the BOLD signal were separable in the young adults but not fully independent in the older adults. Along the valence dimension of neutral and positive it seems possible to dissociate the effects of arousal and valence in the brain, as indicated by this and previous studies (Kensinger and Corkin, 2004; Lewis et al., 2007; Mourão-Miranda et al., 2003), in young adults at least.

An extension of the current paradigm could involve the inclusion of negative emotional stimuli. There is some evidence for example that the processing of highly negative, threatening stimuli is preserved with age (Mather and Knight, 2006; Rösler et al., 2005; Wright et al., 2006), while other studies have found an age-related reduction in negative emotional processing (Mather and Carstensen, 2005). It would be of interest therefore to examine age-related changes in negative emotional processing by isolating the contributory effects of valence and arousal. The use of negative stimuli in a parametric modulation paradigm would however make it difficult to control for the confounding effects of arousal, as the covariation of arousal with absolute valence is particularly strong in the case of negative images (Lang et al., 2008). Perhaps a stimulus set of emotional words might be more appropriate in this case, such as the affective norms for English words (Bradley and Lang, 1999), as it may be more feasible to find negative, lower arousal stimuli when using words rather than images, which tend to elicit a greater emotional response.

In summary, the results of the current study indicate a dissociation in the neural substrates of emotional arousal and valence processing associated with healthy aging. Our results suggest that the aging brain may be less sensitive to the effects of emotional arousal but more responsive to the effects of positive emotional valence. The results also suggest however that older adults may engage in greater emotional regulation in response to highly arousing emotional stimuli, via the activation of the right MFG. It may well be that the neural basis of the "mellow years" lies not so much in reduced processing of negative stimuli, but that attenuated arousal processing is a contributory factor.

\section{Disclosure statement}

None of the authors have any potential or actual conflicts of interest to declare.

The research study had full ethical approval from the St James' Hospital and the Adelaide and Meath Hospital, Incorporating the National Children's Hospital Research Ethics Committee. All of the participants gave written informed consent before commencing the study. 


\section{Acknowledgements}

The authors thank Mr. Sojo Josephs for his assistance in acquiring the neuroimaging data and the Trinity Centre for High Performance Computing (TCHPC) for making computer resources available for the study. E.G.K. was supported by a Ph.D. grant from the Health Research Board (HRB). J.H.B. was supported by a postdoctoral fellowship from the Irish Research Council for Science Engineering and Technology (IRCSET). A.L.W.B. is supported by the Stokes program from Science Foundation Ireland (SFI).

\section{Appendix A. Supplementary data}

Supplementary data associated with this article can be found, in the online version, at http://dx.doi.org/10.1016/ j.neurobiolaging.2012.07.006.

\section{References}

Addis, D.R., Leclerc, C.M., Muscatell, K.A., Kensinger, E.A., 2010. There are age-related changes in neural connectivity during the encoding of positive, but not negative, information. Cortex 46, 425-433.

Anderson, N.D., Iidaka, T., Cabeza, R., Kapur, S., McIntosh, A.R., Craik, F.I., 2000. The effects of divided attention on encoding- and retrievalrelated brain activity: A PET study of younger and older adults. J. Cogn. Neurosci. 12, 775-792.

Beck, A.T., Steer, R.A., Brown, G.K., 1996. Beck Depression Inventory 2nd Edition Manual. Psychological Corporation, San Antonio.

Bradley, B.P., Lang, P.J., 1999. Affective norms for English words. (ANEW): stimuli, instruction manual and affective ratings. Technical report C-1. Center for Research in Psychophysiology, University of Florida, Gainesville, FL.

Bradley, M.M., Codispoti, M., Sabatinelli, D., Lang, P.J., 2001. Emotion and motivation II: sex differences in picture processing. Emotion 1, 300-319.

Bradley, M.M., Sabatinelli, D., Lang, P.J., Fitzsimmons, J.R., King, W., Desai, P., 2003. Activation of the visual cortex in motivated attention. Behav. Neurosci. 117, 369-380.

Braver, T.S., Barch, D.M., 2002. A theory of cognitive control, aging cognition, and neuromodulation. Neurosci. Biobehav. Rev. 26, 809817.

Cahill, L., Haier, R.J., Fallon, J., Alkire, M.T., Tang, C., Keator, D., Wu, J., McGaugh, J.L., 1996. Amygdala activity at encoding correlated with long-term, free recall of emotional information. Proc. Natl. Acad. Sci. U. S. A. $93,8016-8021$.

Cahill, L., Haier, R.J., White, N.S., Fallon, J., Kilpatrick, L., Lawrence, C., Potkin, S.G., Alkire, M.T., 2001. Sex-related difference in amygdala activity during emotionally influenced memory storage. Neurobiol. Learn. Mem. 75, 1-9.

Canli, T., 2004. Functional brain mapping of extraversion and neuroticism: learning from individual differences in emotion processing. J. Pers. 72, $1105-1132$

Canli, T., Zhao, Z., Desmond, J.E., Kang, E., Gross, J., Gabrieli, J.D., 2001. An fMRI study of personality influences on brain reactivity to emotional stimuli. Behav. Neurosci. 115, 33-42.

Carstensen, L.L., Fung, H.H., Charles, S.T., 2003. Socioemotional selectivity theory and the regulation of emotion in the second half of life. Motiv. Emotion 27, 103-123.

Charles, S.T., Mather, M., Carstensen, L.L., 2003. Aging and emotional memory: the forgettable nature of negative images for older adults. J. Exp. Psychol. Gen. 132, 310-324.
Charles, S.T., Reynolds, C.A., Gatz, M., 2001. Age-related differences and change in positive and negative affect over 23 years. J Pers Soc Psychol 80, 136-151.

Choi, H.J., Zilles, K., Mohlberg, H., Schleicher, A., Fink, G.R., Armstrong, E., Amunts, K., 2006. Cytoarchitectonic identification and probabilistic mapping of two distinct areas within the anterior ventral bank of the human intraparietal sulcus. J. Comp. Neurol. 495, 53-69.

Comblain, C., D'Argembeau, A., Van der Linden, M., 2005. Phenomenal characteristics of autobiographical memories for emotional and neutral events in older and younger adults. Exp. Aging Res. 31, 173-189.

Cox, R.W., 1996. AFNI: software for analysis and visualization of functional magnetic resonance neuroimages. Comput. Biomed. Res. 29, $162-173$.

Cremers, H.R., Demenescu, L.R., Aleman, A., Renken, R., van Tol, M.J., van der Wee, N.J., Veltman, D.J., Roelofs, K., 2010. Neuroticism modulates amygdala-prefrontal connectivity in response to negative emotional facial expressions. NeuroImage 49, 963-970.

Davis, M., Whalen, P.J., 2001. The amygdala: vigilance and emotion. Mol. Psychiatry 6, 13-34.

Davis, S.W., Dennis, N.A., Daselaar, S.M., Fleck, M.S., Cabeza, R., 2008. Que PASA? The posterior-anterior shift in aging. Cereb. Cortex 18, 1201-1209.

Denburg, N.L., Buchanan, T.W., Tranel, D., Adolphs, R., 2003. Evidence for preserved emotional memory in normal older persons. Emotion 3, $239-253$.

Dennis, N.A., Daselaar, S., Cabeza, R., 2007. Effects of aging on transient and sustained successful memory encoding activity. Neurobiol. Aging 28, $1749-1758$.

Dolcos, F., LaBar, K.S., Cabeza, R., 2004. Interaction between the amygdala and the medial temporal lobe memory system predicts better memory for emotional events. Neuron $42,855-863$.

Eickhoff, S.B., Stephan, K.E., Mohlberg, H., Grefkes, C., Fink, G.R., Amunts, K., Zilles, K., 2005. A new SPM toolbox for combining probabilistic cytoarchitectonic maps and functional imaging data. NeuroImage 25, 1325-1335.

Eysenck, H.J.E., Eysenck, S.B.G., 1991. Manual of the Eysenck Personality Scales (EPS Adult). Hodder \& Stoughton, London.

Fales, C.L., Barch, D.M., Rundle, M.M., Mintun, M.A., Snyder, A.Z., Cohen, J.D., Mathews, J., Sheline, Y.I., 2008. Altered emotional interference processing in affective and cognitive-control brain circuitry in major depression. Biol. Psychiatry 63, 377-384.

Finnigan, S., O'Connell, R.G., Cummins, T.D., Broughton, M., Robertson, I.H., 2011. ERP measures indicate both attention and working memory encoding decrements in aging. Psychophysiology 48, 601-611.

Fox, E., Russo, R., Bowles, R., Dotton, K., 2001. Do threatening stimuli draw or hold attention in visual attention in subclinical anxiety. J. Exp. Pscyhol. Gen. 130, 681-700.

Gabay, S., Pertzov, Y., Henik, A., 2011. Orienting of attention, pupil size, and the norepinephrine system. Atten. Percept. Psychophys. 73, 123129.

Gavazzeni, J., Wiens, S., Fischer, H., 2008. Age effects to negative arousal differ for self-report and electrodermal activity. Psychophysiology 45, $148-151$

Glisky, E.L., 2007. Changes in cognitive function in human aging, in: Riddle, D.R. (Ed.), Brain Aging: Models, Methods, and Mechanisms. Frontiers in Neuroscience, 5-20. CRC Press, Boca Raton.

Grady, C.L., Bernstein, L.J., Beig, S., Siegenthaler, A.L., 2002. The effects of encoding task on age-related differences in the functional neuroanatomy of face memory. Psychol. Aging 17, 7-23.

Gross, J.J., Carstensen, L.L., Pasupathi, M., Tsai, J., Skorpen, C.G., Hsu, A.Y., 1997. Emotion and aging: experience, expression, and control. Psychol. Aging 12, 590-599.

Grossman, M., Cooke, A., DeVita, C., Alsop, D., Detre, J., Chen, W., Gee, J., 2002. Age-related changes in working memory during sentence comprehension: an fMRI study. NeuroImage 15, 302-317. 
Gruenewald, T.L., Mroczek, D.K., Ryff, C.D., Singer, B.H., 2008. Diverse pathways to positive and negative affect in adulthood and later life: an integrative approach using recursive partitioning. Dev. Psychol. 44, $330-343$.

Grühn, D., Scheibe, S., 2008. Age-related differences in valence and arousal ratings of pictures from the International Affective Picture System (IAPS): do ratings become more extreme with age? Behav. Res. Methods 40, 512-521.

Grühn, D., Smith, J., Baltes, P.B., 2005. No aging bias favoring memory for positive material: evidence from a heterogeneity-homogeneity list paradigm using emotionally toned words. Psychol. Aging 20, 579-588.

Haas, B.W., Constable, R.T., Canli, T., 2008. Stop the sadness: Neuroticism is associated with sustained medial prefrontal cortex response to emotional facial expressions. NeuroImage 42, 385-392.

Hariri, A.R., Bookheimer, S.Y., Mazziotta, J.C., 2000. Modulating emotional responses: effects of a neocortical network on the limbic system. Neuroreport 11, 43-48.

Haxby, J.V., Grady, C.L., Horwitz, B., Ungerleider, L.G., Mishkin, M., Carson, R.E., Herscovitch, P., Schapiro, M.B., Rapoport, S.I., 1991. Dissociation of object and spatial visual processing pathways in human extrastriate cortex. Proc. Natl. Acad. Sci. U. S. A. 88, 1621-1625.

Heinzel, A., Bermpohl, F., Niese, R., Pfennig, A., Pascual-Leone, A., Schlaug, G., Northoff, G., 2005. How do we modulate our emotions? Parametric fMRI reveals cortical midline structures as regions specifically involved in the processing of emotional valences. Cogn. Brain Res. 25, 348-358.

Isaacowitz, D.M., Wadlinger, H.A., Goren, D., Wilson, H.R., 2006. Is there an age-related positivity effect in visual attention? A comparison of two methodologies. Emotion 6, 511-516.

Ishai, A., Ungerleider, L.G., Martin, A., Schouten, J.L., Haxby, J.V., 1999. Distributed representation of objects in the human ventral visual pathway. Proc. Natl. Acad. Sci. U. S. A. 96, 9379-9384.

Kehoe, E.G., Toomey, J.M., Balsters, J.H., Bokde, A.L., 2011. Personality modulates the effects of emotional arousal and valence on brain activation. Soc. Cogn. Affect. Neurosci. doi: 10.1093/scan/nsr059.

Kennedy, Q., Mather, M., Carstensen, L.L., 2004. The role of motivation in the age-related positivity effect in autobiographical memory. Psychol. Sci. 15, 208-214.

Kensinger, E.A., Corkin, S., 2004. Two routes to emotional memory: distinct neural processes for valence and arousal. Proc. Natl. Acad. Sci. U. S. A. 101, 3310-3315.

Kensinger, E.A., Garoff-Eaton, R.J., Schacter, D.L., 2007. How negative emotion enhances the visual specificity of a memory. J. Cogn. Neurosci. 19, 1872-1887.

Kensinger, E.A., Schacter, D.L., 2006. Amygdala activity is associated with the successful encoding of item, but not source, information for positive and negative stimuli. J. Neurosci. 26, 2564-2570.

Kensinger, E.A., Schacter, D.L., 2008. Neural processes supporting young and older adults' emotional memories. J. Cogn. Neurosci. 20, 11611173.

Knight, M., Seymour, T.L., Gaunt, J.T., Baker, C., Nesmith, K., Mather, M., 2007. Aging and goal-directed emotional attention: distraction reverses emotional biases. Emotion 7, 705-714.

Kwon, Y., Scheibe, S., Samanez-Larkin, G.R., Tsai, J.L., Carstensen, L.L., 2009. Replicating the positivity effect in picture memory in Koreans: evidence for cross-cultural generalizability. Psychol. Aging 24, 748754.

Lane, R.D., Reiman, E.M., Bradley, M.M., Lang, P.J., Ahern, G.L., Davidson, R.J., Schwartz, G.E., 1997. Neuroanatomical correlates of pleasant and unpleasant emotion. Neuropsychologia 35, 1437-1444.

Lang, P.J., Bradley, B.P., Cuthbert, B.N., 2008. International Affective Picture System (IAPS): Affective Ratings of Pictures and Instruction Manual. Technical Report A-8. University of Florida, Gainesville, FL.

Lang, P.J., Bradley, M.M., Cuthbert, B.N., 1998. Emotion, motivation, and anxiety: brain mechanisms and psychophysiology. Biol. Psychiatry 44, $1248-1263$.
Leclerc, C.M., Kensinger, E.A., 2008. Effects of age on detection of emotional information. Psychol. Aging 23, 209-215.

Leppänen, J.M., 2006. Emotional information processing in mood disorders: a review of behavioral and neuroimaging findings. Curr. Opin. Psychiatry 19, 34-39.

Lewis, P.A., Critchley, H.D., Rotshtein, P., Dolan, R.J., 2007. Neural correlates of processing valence and arousal in affective words. Cereb. Cortex 17, 742-748.

Liu, W.H., Chan, R.C., Wang, L.Z., Huang, J., Cheung, E.F., Gong, Q.Y., Gollan, J.K., 2011. Deficits in sustaining reward responses in subsyndromal and syndromal major depression. Prog. Neuropsychopharmacol. Biol. Psychiatry 35, 1045-1052.

Mather, M., Canli, T., English, T., Whitfield, S., Wais, P., Ochsner, K., Gabrieli, J.D.E., Carstensen, L.L., 2004. Amygdala responses to emotionally valenced stimuli in older and younger adults. Psychol. Sci. 15, 259-263.

Mather, M., Carstensen, L.L., 2005. Aging and motivated cognition: the positivity effect in attention and memory. Trends Cogn. Sci. 9, 496502.

Mather, M., Knight, M.R., 2006. Angry faces get noticed quickly: threat detection is not impaired among older adults. J. Gerontol. B Psychol. Sci. Soc. Sci. 61, 54-57.

McGaugh, J.L., 2006. Make mild moments memorable: add a little arousal. Trends Cogn. Sci. 10, 345-347.

Morris, J.C., Heyman, A., Mohs, R.C., Hughes, J.P., van Belle, G., Fillenbaum, G., Mellits, E.D., Clark, C., 1989. The Consortium to Establish a Registry for Alzheimer's Disease (CERAD). Part I. Clinical and neuropsychological assessment of Alzheimer's disease. Neurology 39, $1159-1165$.

Morris, J.C., Mohs, R.C., Rogers, H., Fillenbaum, G., Heyman, A., 1988. Consortium to establish a registry for Alzheimer's disease (CERAD) clinical and neuropsychological assessment of Alzheimer's disease. Psychopharmacol. Bull. 24, 641-652.

Mourão-Miranda, J., Volchan, E., Moll, J., de Oliveira-Souza, R., Oliveira, L., Bramati, I., Gattass, R., Pessoa, L., 2003. Contributions of stimulus valence and arousal to visual activation during emotional perception. Neuroimage 20, 1955-1963.

Mroczek, D.K., Kolarz, C.M., 1998. The effect of age on positive and negative affect: a developmental perspective on happiness. J. Pers. Soc. Psychol. 75, 1333-1349.

Murty, V.P., Sambataro, F., Das, S., Tan, H.Y., Callicott, J.H., Goldberg, T.E., Meyer-Lindenberg, A., Weinberger, D.R., Mattay, V.S., 2009. Age-related alterations in simple declarative memory and the effect of negative stimulus valence. J. Cogn. Neurosci. 21, 1920-1933.

O'Neill, W.D., Zimmerman, S., 2000. Neurologial interpretations and the information in the cognitive pupillary response. Methods Inf. Med. 39, $122-124$.

Petersen, R.C., Smith, G.E., Waring, S.C., Ivnik, R.J., Tangalos, E.G., Kokmen, E., 1999. Mild cognitive impairment: clinical characterization and outcome [erratum in 1999;56:760]. Arch. Neurol. 56, 303-308

Phan, K.L., Taylor, S.F., Welsh, R.C., Ho, S.H., Britton, J.C., Liberzon, I., 2004. Neural correlates of individual ratings of emotional salience: a trial-related fMRI study. NeuroImage 21, 768-780.

Pietrini, P., Furey, M.L., Ricciardi, E., Gobbini, M.I., Wu, W.H., Cohen, L., Guazzelli, M., Haxby, J.V., 2004. Beyond sensory images: objectbased representation in the human ventral pathway. Proc. Natl. Acad. Sci. U. S. A. $101,5658-5663$.

Pôrto, W.G., Bertolucci, P.H., Bueno, O.F., 2011. The paradox of age: an analysis of responses by aging Brazilians to International Affective Picture System (IAPS). Rev. Bras. Psiquiatr. 33, 10-15.

Richardson, M.P., Strange, B.A., Dolan, R.J., 2004. Encoding of emotional memories depends on amygdala and hippocampus and their interactions. Nat. Neurosci. 7, 278-285. 
Rösler, A., Ulrich, C., Billino, J., Sterzer, P., Weidauer, S., Bernhardt, T., Steinmetz, H., Frölich, L., Kleinschmidt, A., 2005. Effects of arousing emotional scenes on the distribution of visuospatial attention: changes with aging and early subcortical vascular dementia. J. Neurol. Sci. 229-230, 109-116.

Sharot, T., Phelps, E.A., 2004. How arousal modulates memory: disentangling the effects of attention and retention. Cogn. Affect. Behav. Neurosci. 4, 294-306.

St. Jacques, P.L., Dolcos, F., Cabeza, R., 2009. Effects of aging on functional connectivity of the amygdala for subsequent memory of negative pictures: a network analysis of functional magnetic resonance imaging data. Psychol. Sci. 20, 74-84.

Taylor, S.F., Phan, K.L., Decker, L.R., Liberzon, I., 2003. Subjective rating of emotionally salient stimuli modulates neural activity. NeuroImage $18,650-659$.

Vuilleumier, P., 2005. How brains beware: neural mechanisms of emotional attention. Trends Cogn. Sci. 9, 585-594.

Wager, T.D., Phan, K.L., Liberzon, I., Taylor, S.F., 2003. Valence, gender, and lateralization of functional brain anatomy in emotion: a metaanalysis of findings from neuroimaging. NeuroImage 19, 513-531.
Welsh, K., Butters, N., Hughes, J., Mohs, R., Heyman, A., 1991. Detection of abnormal memory decline in mild cases of Alzheimer's disease using CERAD neuropsychological measures. Arch. Neurol. $48,278-281$.

Welsh, K.A., Butters, N., Hughes, J.P., Mohs, R.C., Heyman, A., 1992. Detection and staging of dementia in Alzheimer's disease. Use of the neuropsychological measures developed for the Consortium to Establish a Registry for Alzheimer's Disease. Arch. Neurol. 49, 448452.

Williams, L.M., Brown, K.J., Palmer, D., Liddell, B.J., Kemp, A.H., Olivieri, G., Peduto, A., Gordon, E., 2006. The mellow years? Neural basis of improving emotional stability over age. J. Neurosci. 26, 64226430.

Wrase, J., Klein, S., Gruesser, S.M., Hermann, D., Flor, H., Mann, K., Braus, D.F., Heinz, A., 2003. Gender differences in the processing of standardized emotional visual stimuli in humans: a functional magnetic resonance imaging study. Neurosci. Lett. 348, 41-45.

Wright, C.I., Wedig, M.M., Williams, D., Rauch, S.L., Albert, M.S., 2006. Novel fearful faces activate the amygdala in healthy young and elderly adults. Neurobiol. Aging 27, 361-374. 